CERN-TH/2001-147

hep-ph/0106049

\title{
Higgs production at hadron colliders in (almost) NNLO QCD "̈
}

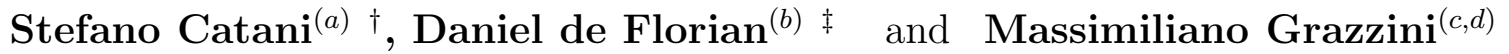 \\ ${ }^{(a)}$ Theory Division, CERN, CH-1211 Geneva 23, Switzerland \\ ${ }^{(b)}$ Institute for Theoretical Physics, ETH-Hönggerberg, CH-8093 Zürich, Switzerland \\ (c) Dipartimento di Fisica, Università di Firenze, I-50125 Florence, Italy \\ ${ }^{(d)}$ INFN, Sezione di Firenze, I-50125 Florence, Italy
}

\begin{abstract}
We compute the soft and virtual NNLO QCD corrections to Higgs production through gluon-gluon fusion at hadron colliders. We present numerical results obtained at the LHC and at the Tevatron Run II.
\end{abstract}

CERN-TH/2001-147

June 2001

${ }^{*}$ Talk given by M. Grazzini at the XXXVIth Rencontres de Moriond, QCD and Hadronic interactions, Les Arc1800, France and at the 9th International Workshop on Deep Inelastic Scattering DIS2001, Bologna, Italy.

$\dagger$ On leave of absence from INFN, Sezione di Firenze, Florence, Italy.

${ }^{\ddagger}$ Partially supported by Fundación Antorchas. 
Although the Higgs boson is a crucial ingredient of the Standard Model (SM), it has so far eluded experimental discovery. LEP results [1] imply a lower limit of $M_{H}>113.5 \mathrm{GeV}$ (at $95 \%$ $\mathrm{CL})$ on the mass $M_{H}$ of the SM Higgs boson, favour a light Higgs boson $\left(M_{H} \lesssim 200 \mathrm{GeV}\right)$ and suggest the observation of signal events at $M_{H} \simeq 115 \mathrm{GeV}$.

After the end of the LEP program, the Higgs search will be carried out at hadron colliders, where the dominant production mechanism is gluon-gluon fusion through a heavy-quark loop. At the LHC [2], $g$ fusion overwhelms the other production channels in the case of a light Higgs boson, and it still provides $\sim 50 \%$ of the total production rate at $M_{H} \simeq 1 \mathrm{TeV}$. At the Tevatron [3], $g g$ fusion gives $\sim 65 \%$ of the total cross section in the mass range $M_{H}=100-200 \mathrm{GeV}$, although this production mechanism leads to an important discovery mode only at $M_{H} \gtrsim 135 \mathrm{GeV}$, when the decay channel $H \rightarrow W W^{*}$ opens up (if $M_{H} \lesssim 135 \mathrm{GeV}$, the decay channel $H \rightarrow b \bar{b}$ suffers from a huge QCD background, and the decay rate $H \rightarrow \gamma \gamma$ is too low to be observed).

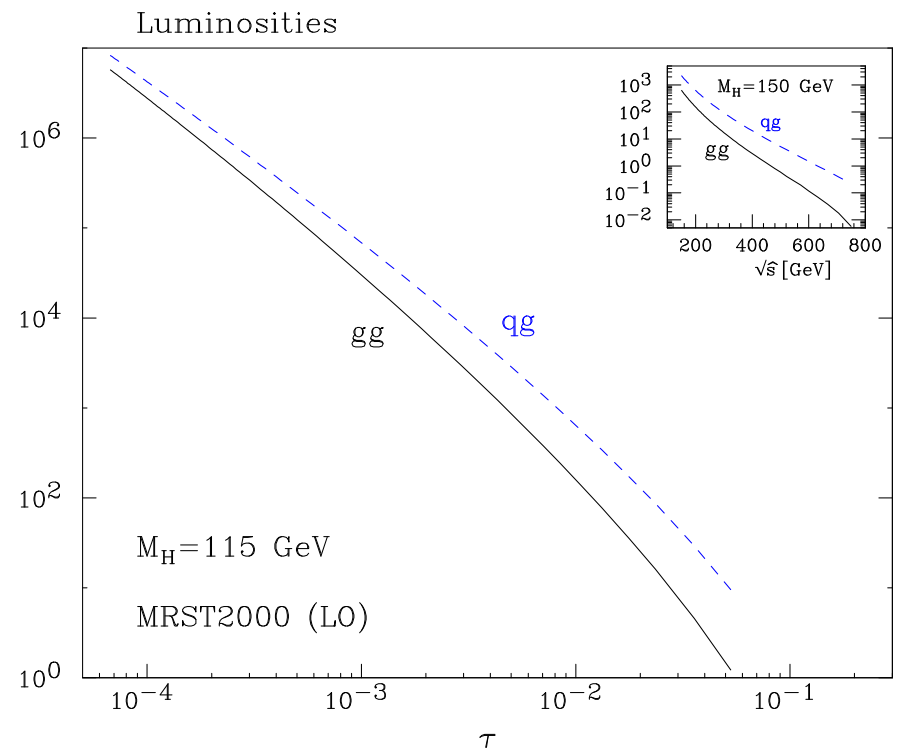

Figure 1: Parton luminosities as a function of $\tau=\hat{s} / s$ at the LHC. The inset plot shows the luminosities at the Tevatron as a function of the partonic centre-of-mass energy $\sqrt{\hat{s}}$.

The NLO QCD corrections to Higgs boson production through $g g$ fusion give a large effect [ $⿴$ ]. Since approximate evaluations [5] of higher-order terms suggest that their effect can still be sizeable, the computation of the NNLO corrections is certainly important.

A first step in this direction has been performed by two groups [6, 7], who have computed the soft and virtual contributions to the NNLO partonic cross section $\hat{\sigma}(g g \rightarrow H+X)$ in the large- $m_{\text {top }}$ approximation. Our calculation [6] was done by combining the recent result [8] for the two-loop amplitude $g g \rightarrow H$ with the soft factorization formulae for tree-level [9] $(g g \rightarrow H g g, H q \bar{q})$ and one-loop [10] $(g g \rightarrow H g)$ amplitudes. The independent calculation of Ref. [7] uses a different method, and the results for the partonic cross section fully agree.

In Ref. [6], we have combined the NNLO partonic cross section with the recent MRST2000 set of parton distributions [11], which includes (approximate) NNLO densities, thus providing a first consistent estimate of the NNLO QCD corrections to the hadronic cross section. In the following, we briefly summarize our main results. 

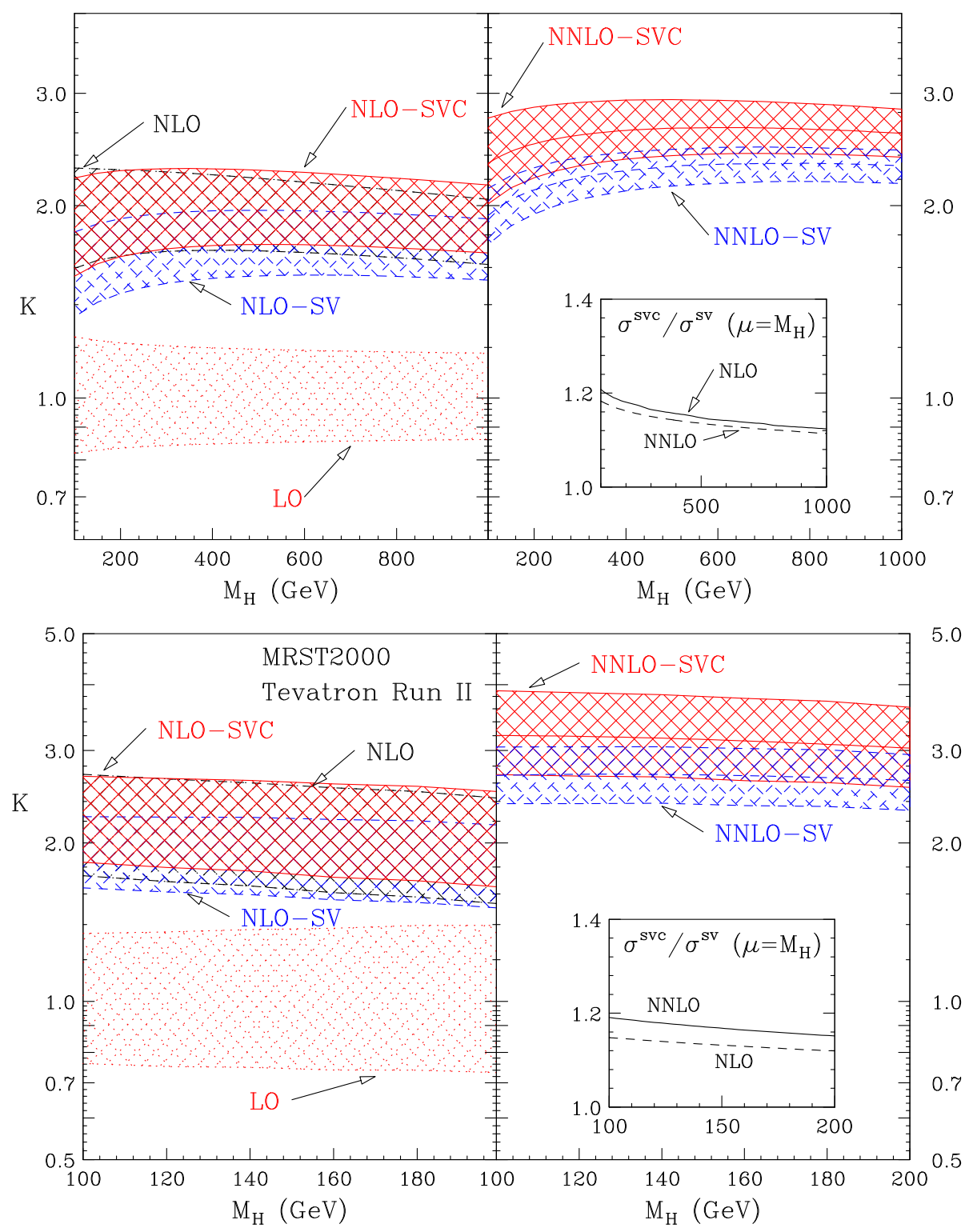

Figure 2: K-factors at the LHC (above) and Tevatron Run II (below): exact NLO result, NLO-SV and NLO-SVC approximations (left), and NNLO-SV and NNLO-SVC approximations (right).

The soft and virtual terms give the most important contributions to the partonic cross section when it is computed at a value of the partonic centre-of-mass energy $\sqrt{\hat{s}}$ that is close to the Higgs boson mass $M_{H}$. The hadronic centre-of-mass energy $\sqrt{s}$ is related to the partonic one as $\hat{s}=\tau s=x_{1} x_{2} s$, where $x_{1}$ and $x_{2}$ are the momentum fractions of the two partons that initiate the partonic subprocess. The density of these partons is controlled by the parton distributions of the colliding hadrons. Since the parton distributions are strongly suppressed at large values of the momentum fractions $x_{1}, x_{2}, \hat{s}$ is typically much smaller than $s$ and, therefore, the soft and virtual terms can approximate the complete result also when the hadronic energy $\sqrt{s}$ is not very close to $M_{H}$. At fixed $M_{H}$, the quantitative reliability of the approximation depends on the value of $\sqrt{s}$ and on the actual value of the parton luminosity. The $g g$ and $(q+\bar{q}) g$ luminosities are shown in Fig. 1. At the LHC, where we use the reference value $M_{H}=115 \mathrm{GeV}$, the parton luminosity decreases by about two orders of magnitude when $\sqrt{\hat{s}}$ increases from $M_{H}$ to $3 M_{H}$ (i.e. when $\tau$ 
increases from $10^{-4}$ to $10^{-3}$ ). At the Tevatron (see the inset plot), the parton luminosity decreases by almost two orders of magnitude when $\sqrt{\hat{s}}$ increases from $\sqrt{\hat{s}}=M_{H}=150 \mathrm{GeV}$ to $300 \mathrm{GeV}$. This observation suggests that the soft and virtual terms can give a good approximation at the LHC and a (slightly) better approximation at the Tevatron Run II.

In Ref. [6], we introduced two approximations, named soft-virtual (SV) and soft-virtualcollinear (SVC), of the partonic cross section. In the SV approximation only the contributions of soft and virtual origin are taken into account. The SVC approximation extends the SV approximation by including the next-to-dominant contribution (which has a collinear origin [5]) in the expansion around the region where $\sqrt{\hat{s}}=M_{H}$.

In the following we present numerical results for the hadronic cross section evaluated up to LO, NLO and NNLO. At the NLO we compare the exact result with those of the SV and SVC approximations. At the NNLO, we add the exact NLO result to our SV and SVC approximations of the NNLO corrections. All the results are presented in term of K-factors, which are defined as the ratio of the hadronic cross section over its LO value. The latter is computed by fixing the factorization and renormalization scales $\mu_{F}$ and $\mu_{R}$ at the central value $\mu_{F}=\mu_{R}=M_{H}$. As a reference, we use the parton distributions of the MRST2000 set, with parton densities and $\alpha_{\mathrm{S}}$ evaluated at each corresponding order.

At the LHC, we find that the results do not strongly depend on the choice of parton distributions: very similar results are obtained by using the CTEQ5 set 12], whereas larger $(\sim 10 \%)$ deviations appear by using the GRV98 set [13]. At the Tevatron Run II, the dependence on the parton distributions is stronger than at the LHC. The CTEQ5 set gives a LO cross section at $M_{H}=100 \mathrm{GeV}\left(M_{H}=200 \mathrm{GeV}\right)$ that is $\sim 10 \%(\sim 30 \%)$ smaller than the value obtained by using the MRST2000 set. Of course, this dependence affects the K-factors through their normalization with respect to the LO cross section.

The K-factors at the LHC and at the Tevatron are shown in Fig. 2. The bands are obtained by independently varying $\mu_{F}$ and $\mu_{R}$ in the range $M_{H} / 2 \leq \mu_{F}, \mu_{R} \leq 2 M_{H}$. The results show a reduction of the scale dependence at NNLO (from about $\pm 20 \%$ at full NLO to about $\pm 10 \%$ and $\pm 15 \%$ at NNLO-SV and NNLO-SVC, respectively).

From the plots on the left-hand side of Fig. 2, we see that at NLO the SV approximation tends to underestimate the exact result, whereas the SVC approximation slightly overestimates it. Therefore, it is reasonable to expect the exact NNLO K-factor to lie inside the corresponding SV and SVC bands. In the case of the production of a light Higgs boson $\left(M_{H}=100-200 \mathrm{GeV}\right)$ at the LHC (Tevatron), this expectation corresponds to $K \simeq 2.2-2.4(K \simeq 3)$, i.e. to an enhancement of the NLO cross section by about 10-25\% (50\%).

Note that, at fixed $M_{H}$, Higgs boson production at the Tevatron is closer to threshold than at the LHC. This implies (see Fig. 2) that the approximation of the fixed-order results in terms of the corresponding SV and SVC contributions works better at the Tevatron than at the LHC. This also implies that, order by order in perturbation theory, QCD radiative corrections are larger at the Tevatron than at the LHC. In particular, QCD contributions beyond NNLO, due to multiple soft-gluon emission, can still be numerically relevant to Higgs boson production at the Tevatron.

\section{Acknowledgments.}

This work was supported in part by the EU Fourth Framework Programme "Training and Mobility 
of Researchers", Network "Quantum Chromodynamics and the Deep Structure of Elementary Particles", contract FMRX-CT98-0194 (DG 12 - MIHT).

\section{References}

[1] See the contributions by A.N. Okpara hep-ph/0105151, T. Kawamoto hep-ex/0105032 and P. Garcia-Abia hep-ex/0105034 at this Conference, and references therein.

[2] CMS Coll., Technical Proposal, report CERN/LHCC/94-38 (1994); ATLAS Coll., ATLAS Detector and Physics Performance: Technical Design Report, Volume 2, report CERN/LHCC/99-15 (1999).

[3] M. Carena et al., Report of the Tevatron Higgs working group, hep-ph/0010338.

[4] S. Dawson, Nucl. Phys. B 359 (1991) 283; A. Djouadi, M. Spira and P. M. Zerwas, Phys. Lett. B 264 (1991) 440; M. Spira, A. Djouadi, D. Graudenz and P. M. Zerwas, Nucl. Phys. B 453 (1995) 17.

[5] M. Kramer, E. Laenen and M. Spira, Nucl. Phys. B 511 (1998) 523.

[6] S. Catani, D. de Florian and M. Grazzini, JHEP 0105 (2001) 025.

[7] R. V. Harlander and W. B. Kilgore, hep-ph/0102241.

[8] R. V. Harlander, Phys. Lett. B 492 (2000) 74.

[9] J. M. Campbell and E. W. Glover, Nucl. Phys. B 527 (1998) 264; S. Catani and M. Grazzini, Nucl. Phys. B 570 (2000) 287.

[10] Z. Bern, V. Del Duca, W. B. Kilgore and C. R. Schmidt, Phys. Rev. D 60 (1999) 116001; S. Catani and M. Grazzini, Nucl. Phys. B 591 (2000) 435.

[11] A. D. Martin, R. G. Roberts, W. J. Stirling and R. S. Thorne, Eur. Phys. J. C 18 (2000) 117.

[12] H. L. Lai et al. [CTEQ Collaboration], Eur. Phys. J. C 12 (2000) 375.

[13] M. Gluck, E. Reya and A. Vogt, Eur. Phys. J. C 5 (1998) 461. 